\title{
Muscle tear: innovation in the classification and imagenological approach of sports muscle injuries
}

\begin{abstract}
The muscular system provides support and movement due to the muscles that compose it (cardiac, striated and smooth), through muscular contraction, which is divided into slow (repetitive) and fast (for high intensity and resistance), also by Isometric and isotonic tension (eccentric and concentric). Muscle injuries (tears) are related to the practice of physical and sports activity, which can occur at any time due to bruising (trauma) or bloating (hyperextension), and when they occur they generate edema, ecchymosis - hematoma, functional impotence and deformation in its architecture. Timely management as well as classification and optimal imaging approach allow an improvement in therapeutics recovery and return to physical activity - competition of the patient.
\end{abstract}

Volume 4 Issue 6 - 2020

\author{
Alexandra Alvarez Tercero, Ricardo Vargas \\ Fernandez, Irene Gutierrez Murillo \\ Department of Medicine, UNIBE, Costa Rica
}

Correspondence: Alexandra Alvarez Tercero, Department of Medicine, UNIBE, Costa Rica, Tel 506 83I3294I,

Email Egipt2003@gmail.com

Received: June 17, 2020 | Published: December 31, 2020

Keywords: skeletal muscle fibers, skeletal muscle, magnetic resonance imaging, ultrasonography, muscle injuries, classification of muscle injuries in sports, muscle injuries in athletes, imaging classification of muscle injuries and treatment of muscle injuries

\section{Introduction}

The muscular system is in charge of providing support - body movement, and is made up of more than 650 muscles, which are divided into 3 categories (cardiac, smooth and striated). The muscles are divided into $10 \%$ smooth and cardiac muscle, and $40 \%$ skeletal or striated muscle, which in turn is made up of numerous fibers with a diameter of 10-80 $\mu \mathrm{m}$ made up of smaller subunits. Each muscle fiber has a membrane, sarcomere, bands and a disk; and it is made up of thousands of myofibrils (1500 myosin filaments and approximately 3000 actin filaments), adjacent to each other, being large polymerized protein molecules responsible for muscle contraction. These filaments partially interdigitate, thus giving alternate, light (actin filaments known as bands I, isotropic in polarized light) and dark (myosin filaments or A bands, anisotropic in polarized light) overlapping each other and with projections. (Crossed bridges), giving an interaction that causes muscle contraction. ${ }^{1-5}$ The muscular contraction is divided according to the contractile function of the fiber, in type I or slow contraction, which is repetitive, resistant to fatigue, with a greater amount of mitochondria and capillary action, ideal for low intensity exercise; and type II or rapid contraction, for high intensity and short time activity, with high resistance, capable of producing greater force.

It is also divided according to the tension into isometric or without change in length, and isotonic, that is, from tension associated with the change in fiber length, which in turn is subdivided into concentric (fiber shortening) and eccentric (elongation of fiber). ${ }^{1-5}$ Muscle tears or injuries are generally related to the practice of physical and sports activity - competitive, however these can occur at any time or daily activity. A muscle tear or injury is the loss of the continuity of the muscle tissue or its connective tissue, that is, a traumatic injury to the interior of the muscle, which consists of a laceration of greater or lesser number of muscle fibers (myofibrils) that break, can bleed and cause bleeding. ${ }^{1-5}$ The injury can be caused by contusion (trauma) or distension (hyperextension during contraction) of the muscle fibers responsible for muscle contraction, as well as by the connective tissue that surrounds them and can present with symptoms such as even acute localized pain, accompanied by inflammation, ecchymosis / hematoma, functional impotence and structural deformation. ${ }^{1-5}$ The objective of this bibliography review is to describe the types of muscle tears and the phases of recovery from muscle injuries for the care of patients who carry out light-moderate physical activity as well as elite athletes, as well as to inform the innovation of the classification and approach updated imaging for better therapy.

\section{Methods}

Bibliographic review is performed by searching Google Academic, NCBI databases; PMC and sports federations (football clubs, confederations) to obtain research material, using. The reviews in each document were read, which allowed determining their use for this review, and 3 books, 1 video and 13 articles were obtained in their entirety. Publications of extensive and detailed material from the multiple classifications, approach and treatment of muscle injuries were included, as well as little detailed publications from the approach and classification of muscle injuries were excluded.

\section{Physiology of muscle contraction}

The muscle fiber is made up of multiple myofibrils (made up of multiple sarcomeres, which is the contractile unit of the striated muscle). The sarcomere is made up of thick filaments (myosin exclusively) and thin filaments (tropomyosin, troponin, and actin), which in turn makes up various structures such as the M line (being the central structure of each fiber), $\mathrm{H}$ band (thick filaments only), band A (both filaments: actin and myosin), band I (thin filaments only) and line $\mathrm{Z}$ (disk Z, which is the anchoring zone for actins); in turn between $\mathrm{Z}$ lines is titin, the largest protein in the human body. ${ }^{1-6}$ Muscle contraction is due to the interaction of multiple structures, the thin filament (the actin enveloped by tropomyosin and the sites of action are covered by troponin and its subunits) and the thick filament 
(myosin covered by ADP molecules; the which will keep it stable and firm without contracting, an action that they possess for themselves, prepared for contraction). ${ }^{1-6}$

To activate the filaments, the membrane action potential must be given by stimulation of acetylcholine from the alpha motor nerone, by voltage-dependent sodium channels, reaching the threshold and the polarity change being unleashed, allowing the opening of channels (mainly tubule $\mathrm{T}$, closest site to the sarcoplasmic reticulum) where the dihydropyridine receptor (voltage-dependent calcium channel) is located and this allows the entry of calcium. ${ }^{1-6}$ With the entry of calcium and its interaction with the sarcoplasmic reticulum, intracellular calcium is released, which seeks to bind to the c subunit of troponin and release the actin action site, which in turn releases the site of action of myosin, to join it and finally generate the contraction..$^{1-6}$ With muscle contraction, ATP is required for re-entry of intracellular calcium into the reticulum. This is given by the SERCA pump of the reticular membrane, by means of an opposite concentration gradient (attracts calcium to its interior), releasing ATP for its binding again to myosin and becoming ADP. In turn, the c subunit stops receiving the calcium stimulus and the troponin covers the actin action site again, returning to the initial stage. ${ }^{1-6}$

\section{Pathological anatomy: phases of muscle regeneration}

Knowing the phases by which the muscular injury develops when the trauma occurs or an alteration in the adequate contraction of the same, helps in a better recovery of the injury caused, because the therapy can positively or negatively affect this process. ${ }^{7-9}$ It should be noted that muscle tissue is very dynamic and therefore can be affected by external factors of its environment as well as positive stimuli to it, that is, it is manipulable by mechanical processes such as massages, electromagnetic fields as well as chemical processes (use of NSAIDs). Furthermore, it is a source of different types of somatic stem cells, myogenic stem cells (here are the satellite cells: main responsible for muscle growth, muscle regeneration) and multipotent muscle derived stem cells. ${ }^{7-9}$ The regeneration capacity of the muscles decreases with age, while fibrosis increases, and it is known that the repair of a muscle injury does not tend to be uniform or homogeneous, therefore, it is affected with passage of time (Table 1) \& (Figure 1). ${ }^{7-9}$ Recovery period chronology and the components that interfere in each period of time. Source: own elaboration based on Pedreta: Muscle injuries in sport. ${ }^{7}$

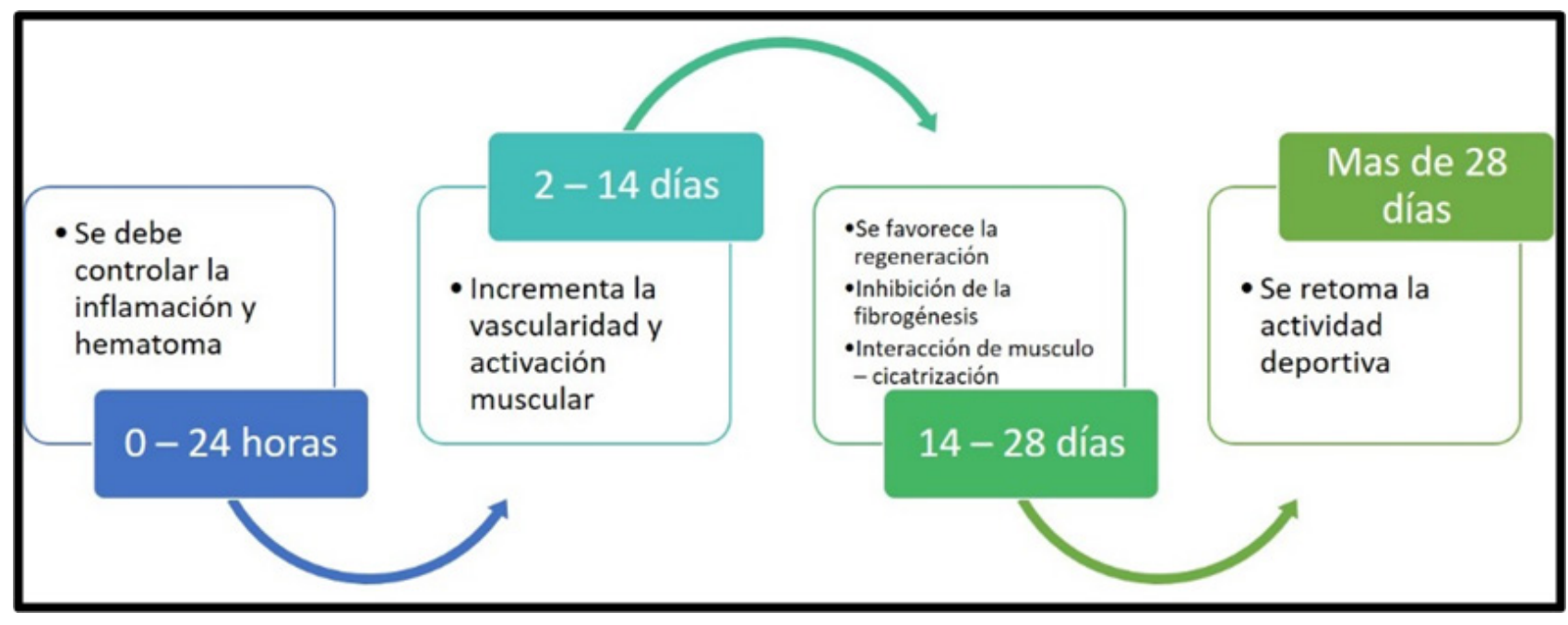

Figure I Own elaboration based on Pedreta: Muscle injuries in sports.

Table I Phases of muscle regeneration

\begin{abstract}
It covers the period after physical activity (first 24-48 hours). A hematoma is evidenced by the extravasation of blood from the vessels with rupture, release of cytosines and fibroblasts, stimulation of various cells (macrophages, neutrophils Acute Phase and lymphocytes).A degenerative effect occurs due to the activation of these cells together with inflammation on the myofibrillar proteins and simultaneously vasodilation-angiogenesis promoted by factor $D$ and vascular growth factor. The objective of these two elements (factor $D$ and vascular growth factor) is to generate adequate vascularity to the place, improving the cellular metabolic state with greater oxygenation.
\end{abstract}

The main function of the growth factor and other substances (mainly fibroblastic growth factor) involved is the activation - stimulation of the muscle satellite cells, with the aim of triggering the cascade of muscle regeneration, arteriogenesis and myogenesis in the damaged muscle. The differentiation of satellite cells is due to the various factors that intervene in this phase and at this point we must specifically try to provide adequate treatment because it influences fibrotic repair muscle regeneration

Regeneration Phase

Fibrogenesis

It occurs in a period of 14-28 days where there is greater activity in the cell lines of muscle regeneration and decreases Phase differentiation, so that in therapeutics, multiple drugs have inhibited fibrogenesis (allows better cell differentiation muscle precursors) and growth factor beta I, improving muscle regeneration

Source: own elaboration based on Pedreta: Muscle injuries in sport; Executioner: Ultrasonographic classification of muscle tears and Muñoz: Sports muscle injuries $^{7-9}$ 


\section{Tear classification: anatomic criteria}

The criteria that provide the classification of the types of muscle injury (fiber tears) by anatomical injury, mechanism of injury and image visualization should be separated, since each category has various key components for its identification (Table 2). ${ }^{8}$ The classification by anatomical criteria, provides a comprehensive visualization of the muscle injury, while the classification by image criteria, provides a common language in the medical field for a generalized approach to a simple but at the same time complex picture such as the injury of the muscle tissue, as it is a dynamic complex (Table 3). ${ }^{7,8}$ Image of each type of tear (ultrasound).

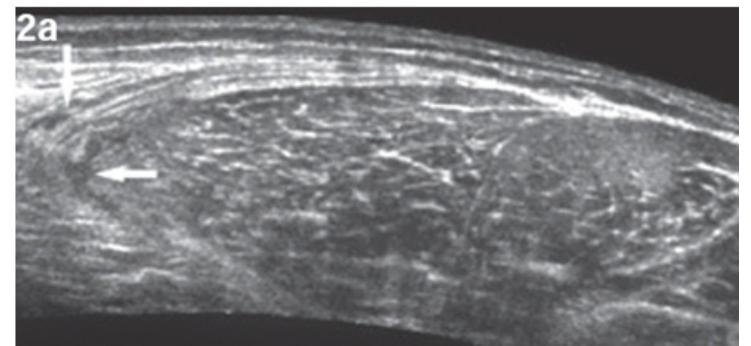

Myofascial tear.Thickening and erasure of the external contour with thin (laminar) bands of blood fluid

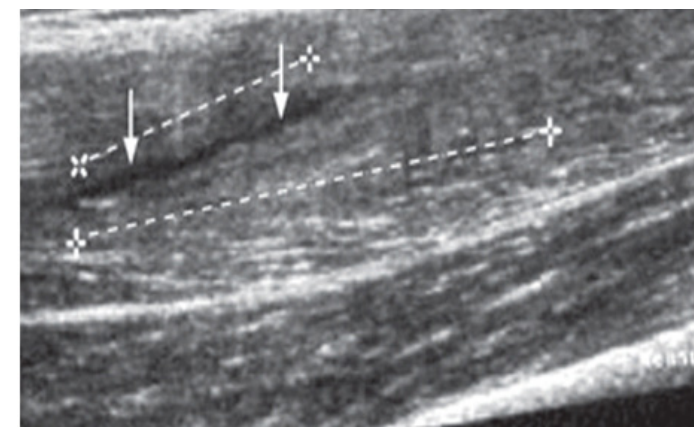

Fibrillar tear. Compound injury

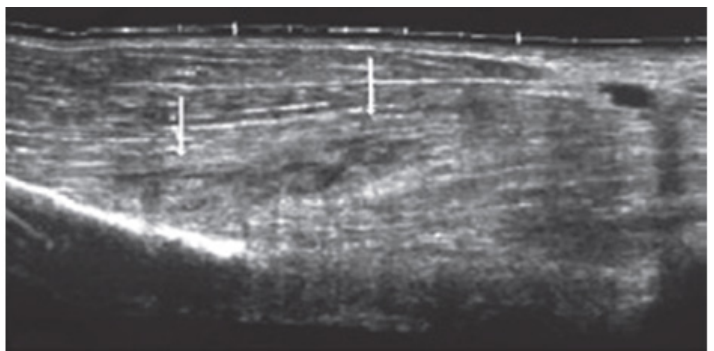

Multifibrillary tear. Multiple linear or fibrillary lesions and extensive edema

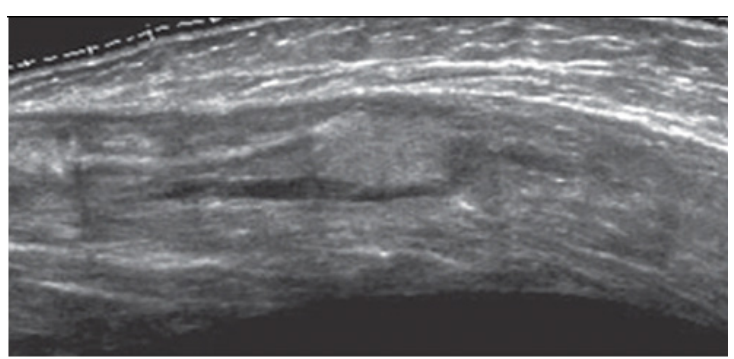

Fascicular tear. $2 \mathrm{~mm}$ thick lesion, $7 \mathrm{~cm}$ long. Distortion of the surrounding muscle pattern by edema

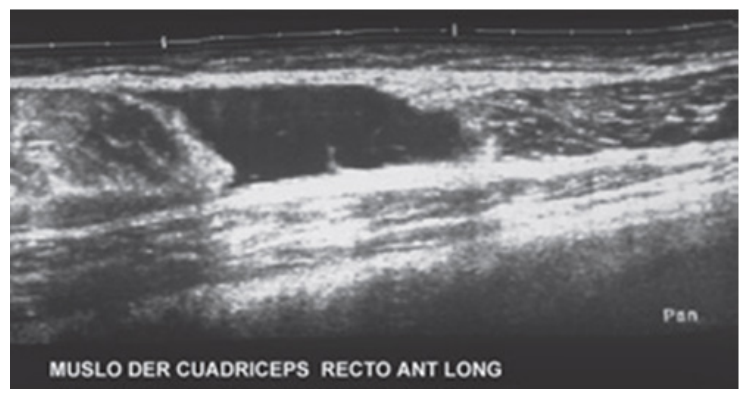

Total tear. Has disruption and hematoma (echogenic in acute phase and anechogenic in chronic phase)

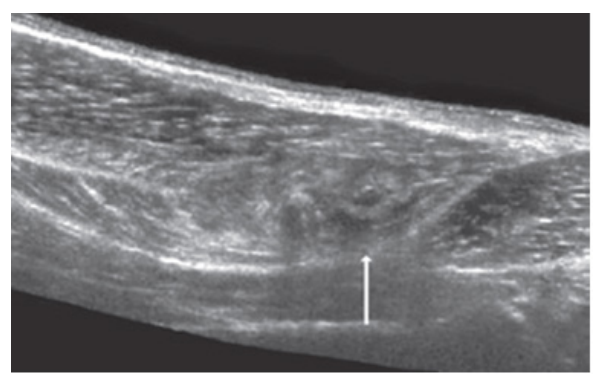

Adherenciolysis. Marginal redesgarro with scar in evolution Wide area of alteration due to previous tear.

Source: own elaboration based on Verdugo: Ultrasonographic classification of muscle tears ${ }^{8}$

Table 2 Anatomical classification with ultrasound image data
Myofascial tear
- It comprises two anatomical elements, the fascia and the peripheral muscle fibers and they are generally good prognosis lesions and they usually heal without sequelae in a short time of 15 to 25 days.
- It is the most frequent tear in high-performance athletes and can easily recur (more frequent are hamstrings, biceps femoris, triceps surae)
Image:The muscle fascia loses its sharp contour and is accompanied by hypercogenic fluid laminar bands with some retraction of the peripheral muscle fibers. There is always peripheral edema of muscle fibers that is seen as hypercogenic areas.


Table Continued..

\begin{tabular}{|c|c|}
\hline \multirow[t]{3}{*}{ Fibrillary tear } & $\begin{array}{l}\text {-Very fine linear type lesion, it has a variable length but with a thickness that should not exceed } 2 \mathrm{~mm} \text { (it occurs in } \\
\text { the thickness of the musculature, and is accompanied by perilesional edema of variable amount), it heals without } \\
\text { sequelae and is of good prognosis. }\end{array}$ \\
\hline & $\begin{array}{l}\text { - It occurs more frequently in high-performance athletes, affects any muscle, but is more frequent in the hamstrings } \\
\text { and quadriceps, rare in the triceps surae. }\end{array}$ \\
\hline & $\begin{array}{l}\text { - Image: fine hypoechoic defects in a context of surrounding hyperechoic edema. They are usually small lesions, not } \\
\text { more than } 3 \text { to } 4 \mathrm{~cm} \text { in length. }\end{array}$ \\
\hline \multirow[t]{3}{*}{ Multifibrillary tear } & $\begin{array}{l}\text { Variant of the previous one, it has greater clinical importance, it consists of several linear continuity solutions, } \\
\text { generating a larger lesion focus, always associated with edema of greater extension and intensity. Its prognosis is } \\
\text { still good, but it requires a longer healing time (they can leave small retractable scars). }\end{array}$ \\
\hline & $\begin{array}{l}\text {-They are generally seen in high performance athletes and amateurs. Probably the most common site is the } \\
\text { anterior rectum of the quadriceps. }\end{array}$ \\
\hline & $\begin{array}{l}\text { - Image: patterns similar to fibrillar tear, with the difference that it presents with several hypoechogenic linear } \\
\text { defects, with a greater degree of edema. }\end{array}$ \\
\hline \multirow[t]{2}{*}{ Fascicular tear } & $\begin{array}{l}\text { - Lesion of greater importance and can occur in the thickness / periphery of the muscle, where it is accompanied } \\
\text { by compromise of the fascia, with a blood collection associated with the appearance of ecchymosis. }\end{array}$ \\
\hline & $\begin{array}{l}\text { - Image: well-defined muscle with a hypoechoic defect } 3 \text { or more centimeters thick and of variable length. The bed } \\
\text { of the break is accompanied by a hematic collection that may occasionally be larger due to an expansive effect. }\end{array}$ \\
\hline \multirow[t]{2}{*}{ Total tear } & $\begin{array}{l}\text {-All serious injuries that will leave some degree of loss of function, muscle imbalances and large scars. They include } \\
\text { from a thick segment to the entire thickness of the muscle and here also include complete tendomuscular tears } \\
\text { and those that are accompanied by avulsion of the bone insertions, a situation that can occur in the direct tendon } \\
\text { of the anterior rectum of the quadriceps and at the origin of hamstring tendons. }\end{array}$ \\
\hline & $\begin{array}{l}\text { - Image: Generally they are accompanied by hematomas of a certain magnitude and extensive ecchymoses. The } \\
\text { hematoma is echogenic and gradually loses its echogenicity until it becomes anechogenic in the chronic phase. }\end{array}$ \\
\hline \multirow[t]{2}{*}{ Adherenciolysis } & $\begin{array}{l}\text { Refers to re-tearing or opening of the scar, which causes local pain. It can be partial and commonly occurs on the } \\
\text { periphery of the lesion. It occurs due to the lack of rest (less than established), so the recurrent injury is greater } \\
\text { than the original one. }\end{array}$ \\
\hline & - Image: Subtle sheets of fluid in relation to foci of scarring. Similar to myofascial tear. \\
\hline
\end{tabular}

Source: own elaboration based on Verdugo: Ultrasonographic classification of muscle tears ${ }^{8}$

Table 3 Classification by image criteria

\begin{abstract}
Tear Grade I · It is a muscle strain, that is, when the muscle is stretched to its maximum limit. They are microscopic lesions with a compromise (mild - benign) of less than $5 \%$ of the muscle.
\end{abstract}

- His clinic is severe pain without determining a precise point of greater sensitivity, and is indistinguishable from a muscle cramp.

- It has a formation of small serohematic liquid cavities that fill the vacuum produced by the myofibrillar retraction zones subsequent to the micro-rupture (it may be surrounded by edema).

- Difficult to diagnose sonographically because they are small, but the cavities are shaped like a "flame" and are seen in hypoechogenic areas within the muscular belly.

Tear Grade II · It is a more extensive lesion, where the elongation went beyond the maximum limit of its elasticity with a commitment greater than $5 \%$ of the thickness.

(moderate - $\quad$ At the time of the injury, a "jerk" is experienced accompanied by intense local pain of sudden onset. It reaches total muscle partial) impotence and muscle function slowly recovers in the following days. There is localized pain on palpation and increased volume. If the muscle is superficial, ecchymosis may be found distal to the lesion.

- Echographically, it is based on the appearance of a discontinuity zone with disruption of the fibro-fatty septa, where a gap between fibers occupied by a hematoma is identified. Fragments of the ruptured muscle emerging from the walls of the cavity can be identified within the bruise occupying the rupture. The pressure exerted with the transducer demonstrates how these fragments float freely. Compromise of the entire muscular belly, with total separation of the retraction ends and interposition of hematomas (seal of
Tear Grade III muscle rupture, and its magnitude indicates the extent of the injury. It may be intermuscular, characterized by the presence of blood that dissects the fascial planes between the muscles).

- He has ecchymosis with a palpable structural defect, and sonographically when hematomas are present it can be diffusely hypercogenic. As the hours pass, the sacred behaves as a homogeneous and hypercogenic mass. Then the blood elements tend (severe - total) to separate giving a liquid-liquid level.A characteristic finding of a complete tear is the presence of fluid in the epimysium surrounding the entire circumference of the muscle. Days later, this collection becomes uniformly anechogenic, which is reabsorbed in a variable time and dependent on the initial magnitude of the injury.

Source: Own elaboration based on Soto: Ultrasound Classification of Muscle Tears; Executioner: Ultrasonographic classification of muscle tears, and Muñoz: Sports muscle injuries: Diagnostic imaging ${ }^{3,8,10}$ 


\section{Tear classification: image criteria}

The classification by anatomical criteria provides a more detailed classification of the muscle injury, the classification by image criteria is a way to simplify the characterization of the muscle injury, excluding size and prognostic factor, which is why in certain communities of the medical union, it tends to be difficult to accept, more used for its ease. This classification takes into account the degree of preparation of the individual, qualitatively the tear (prognosis and recovery time) and measurement of the injury (quantitatively); This means an approximation but not an exact data. ${ }^{3,8}$ With it, it is a matter of unifying criteria for greater facilitation of understanding among related personnel. This classification excludes lacerations or injuries caused by external agents, and even tear-simulating injuries (delayed muscle pain syndromes, compartment syndrome, pathologies due to muscle overload) (Table 4). ${ }^{3,8}$

Table 4 Mechanism of muscle injury

Indirect or intrinsic
(elongation, late onset or
compartment syndrome)

Direct or extrinsic (contusion and laceration)

-They are manifested by stretching the muscle, by applying a greater tensional force to the resistance of the tissue, when it is in active (eccentric) contraction, that is, an elongation resulting from an intrinsic force generated by a sudden contraction of the muscle.

- It is related to some sports such as gymnastics, athletics, weightlifting, soccer has a greater predisposition to these injuries. The muscles most affected are the muscles of the lower extremities, biceps femoris, anterior rectum, triceps surae. The patient reports sudden pain, such as a "pull or prick."

-The most elementary lesion is edema, a translation of focus of distension without fibrillar rupture (contracture). The excruciating, lacerating pain in the affected muscle region decreases with rest but worsens when the muscle contracts.

-The limb appears swollen, but in cases of being superficial, that is, tears of the superficial muscles, a cutaneous hematoma may appear (generally approximately within 24 hours post trauma) and the area of the hematoma should not be confused with the lesion because the blood dissects inferiorly to the traumatized area. Functional importance is proportional to the extent of the injury, and complete rupture translates into total functional impotence in the area of the affected muscle.

-They are related to direct contusion, either with an object or during the performance of physical activity in teams such as soccer, and corresponds to the result of the impact of the muscle mass against a hard surface or bone; the muscle fibers remain compresses along with the corresponding vessels and a hematoma is formed. ${ }^{8-11}$

- Sometimes it also involves fascia, so the hematoma can also cover both structures (fascia and muscular belly). ${ }^{8-11}$

- On ultrasound it is seen as cavities with irregular edges, and direct trauma causes a contusion of the fibro-fatty septa, these are thickened and diffuse bleeding causes increased echogenicity. ${ }^{8-11}$

- Muscle laceration is the result of penetrating injury, a manifestation of polytraumatized patients

Source of own elaboration based on Verdugo: Ultrasonographic classification of muscle tears; Muñoz: Sports muscle injuries (both writings); and Medical Services of Futbol Club Barcelona: Guide to the clinical practice of muscle injuries. ${ }^{8-11}$

\section{Injury mechanisms}

The mechanisms involved in the formation of a muscle injury are due to the intervention of intrinsic and indirect factors (muscle properties) and extrinsic factors - direct on the surface (due to trauma, laceration) (Table 5). ${ }^{8-11}$

Table 5 Evolution of injury (chronology)

Ummediately $\quad$ Upon injury, a patient-directed survey is conducted. When there is no major rupture, early diagnosis is not easy, so it
is important to wait for it to evolve and thus carry out additional tests.

At 12 o'clock

Ultrasonography does not allow an accurate diagnosis in mild muscle injuries, but it does from second degree injuries. Minor lesions can be determined with serum myosin protein that should not be present in the blood.

At 24 hours

Ideal time for an MRI diagnosis with an adequate prognosis.

At 48 hours

Ideal time to establish an adequate diagnosis and prognosis by means of ultrasound.

Source: own elaboration based on Futbol Club Barcelona Medical Services: Guide to clinical practice of muscle injuries - An objective classification for muscle injuries, and Valle: Muscle Injuries in Sports ${ }^{11-13}$

\section{Risk factor's}

Multiple external factors intervene in promoting a muscle injury, increasing the risk of it happening as well as complications of this pathology. They can be classified into intrinsic risk factors (age, sex, body composition, genetic predisposition, medical history of previous injuries, general condition), that is, those related to the patient (morbidities) and extrinsic factors (Error when training, material that is uses the environment, psychological conditions, season, toxic habits) or totally external to it, increasing the incidence of injury. Factors that are not part of the classification must also be taken into account, but rather are components developed during physical activity such as alterations in the mechanical axes of the extremities (irregular support), support zone (coordination and footfall), muscle balance coordination (execution of movement), muscle fatigue and degree of elasticity. 


\section{Frequency and location of injuries}

The frequency of the appearance of injuries can be estimated in $31 \%$ of the cases of athletes, while the relapse is estimated between $14-32 \%$, depending on the type of sport performed; however, these data can be modified by the location and incidence of the injury. The location tends to associate mostly the lower limbs (preferably thigh) due to the large number of muscles that make up this anatomical area, the hamstrings and quadriceps being the most affected. The abductors and posterior leg region are followed. ${ }^{7}$

\section{Clinical manifestations}

The muscle tear or injury has variations in its presentation, which vary in intensity in each patient and depends on its severity. Associated symptoms include: spontaneous or sudden onset pain, tenderness, and functional impotence; These may appear in the first 12-24 hours after exercise and in some cases tend to resolve spontaneously slowly as days go by. ${ }^{7}$ Among the signs that a patient may manifest, pain on palpation, pain in a relaxed state, is the hematoma called the seal of the injury of a muscle fiber, which can vary in location, where it is more frequent intermuscular characterized by the presence of blood that runs between the muscle fascial planes. Ultrasonographically, it tends to vary if it is active or recent (diffusely hyperechogenic), and after a period of time it becomes a homogeneous and hypogenic mass, and even when the blood elements are separated, there is a liquidliquid level. As the days go by, this image will be from a uniform anechoic collection that can be absorbable (depending on the severity and extent of the injury). ${ }^{10}$ However, when it varies, there may be cases where the trauma is minimal that the patient does not perceive and has spontaneous resolution, where it occurs with discomfort at the end of physical activity or in the next 48 hours, without major affectation. It should be remembered that the lesions with the worst prognosis are those where the muscle is in a state of relaxation. ${ }^{7}$

\section{Diagnosis - imagenological approach}

For muscle injuries, their diagnosis is made clinically, that is, with a medical history and physical examination, so the anamnesis must contain detailed information supplemented by a medical physical examination. These elements are continuously supported by ultrasound studies and magnetic resonance imaging, ideally, because a plain radiograph shows calcifications, it could even be said that it visualizes a bit of an increase in muscle volume and fat fascia but it is not very useful, and computed axial tomography has limited use, and is supportive of plain radiography when it detects calcifications. ${ }^{11-13}$ In the anamnesis it must contain local and general antecedents (similar and previous events, if there was pharmacological treatment), description of the injury moment (such as the event, moment of manifestation: before, during or after the activity), immediate evolution (continuity of the activity or abandonment of it, evolution of pain) ${ }^{11-13}$ During the physical examination, 4 aspects must be taken into account: inspection (ecchymosis or deformities), palpation (painful points or muscle spasms), requesting the active contraction of the affected muscle, first with the muscle with stretching, more sensitive in minor injuries and then with manual resistance (observe whether it is possible, whether or not it is painful and the type of contraction), and assess the analytical flexibility of the muscle (pain on passive stretching). ${ }^{11-13}$

As the evolution of the lesion occurs, the first procedure that is performed immediately is the medical history with physical examination. 12 hours after this stage, a physical examination is performed again together with ultrasound and biochemical markers. After 24 hours, the patient underwent a physical examination, ultrasound, magnetic resonance imaging with biochemical markers, and after 48 hours, only an ultrasound with physical examination was performed (Table 6). ${ }^{11-13}$ Within the approach, the type of injury, its evolution and the approach available must be taken into account. On multiple occasions, the variation in the use of one imaging technique or another differs in how the picture presented by the patient (athlete elite athlete) will be approached in the end. For a long time, ultrasoundultrasound criteria based on histopathological and imaging evidence (ultrasound and magnetic resonance imaging) have been used, thus limiting the adequate reading of highly dynamic tissue. ${ }^{9,11-13}$

Table 6 Ultrasound imaging criteria

\begin{tabular}{lll}
\hline 0 & Contracture and DOMS & Inconsistent signs, edema between fibers and myofascial, with increased local vascularity. \\
one & Fibrillary microcrack / muscle elongation & Minimal discontinuity solution, edema between fibers and interfascial fluid (indirect sign) \\
2 & Fibrillary rupture & Clear muscle defect, interfascial fluid and hematoma. \\
3 & Muscle tear & $\begin{array}{l}\text { Complete muscle / tendon disruption, with retraction of the uninserted portion of the } \\
\text { muscle (evident stump). }\end{array}$
\end{tabular}

DOMS: Delayed onset muscle soreness or late-onset muscle pain

Source: own elaboration based on Futbol Club Barcelona Medical Services: Guide to clinical practice of muscle injuries - An objective classification for muscle injuries, and Valle: Muscle Injuries in Sports ${ }^{11-13}$

\section{Imaging criteria: ultrasound/ultrasound}

It is a dynamic study that complements the clinical examination, that is, an evolutionary follow-up study. The guided evacuation of cavities and complemented with painful echo palpation of the determined muscle (previously identified by ultrasound) is of great help for topographic diagnosis (Table 7) ${ }^{11-13}$

\section{Imaging criteria: magnetic resonance}

It is a high sensitivity and precision imaging study to identify the affected structure (Table 8). ${ }^{11-13}$ Currently, a technique for evaluating "muscle tone" called tensiomyography has been proposed, but there is little scientific evidence for this methodology. ${ }^{11-13}$ 
Table 7 Magnetic resonance imaging criteria

\begin{tabular}{lll}
\hline 0 & Contracture and DOMS & Interstitial and intramuscular edema, increased T2 signal, and fat suppression sequences. \\
one & Fibrillary microcrack / muscle elongation & Increased interstitial and slightly intermuscular signal. \\
2 & Fibrillary rupture & Lots of interstitial signal, focal muscle defect, increased signal around the tendon. \\
3 & Muscle tear & Complete muscle / tendon disruption, with retraction \\
DOMS: Delayed onset muscle soreness or late-onset muscle pain
\end{tabular}

Source: own elaboration based on Futbol Club Barcelona Medical Services: Guide to clinical practice of muscle injuries - An objective classification for muscle injuries, and Valle: Muscle Injuries in Sports ${ }^{11-13}$

Table 8 Current classification units
Mechanism (M) Direct (contusion, better prognosis) or indirect (stretch or sprint) injury
Location (L)
Located in the proximal, middle, or distal third of the muscle. It is related to the myotendinous junction (proximal or distal) and the closer to the tendon, the worse prognosis it will have.
Severity Degree It is numbered from 0 to 3, under the criterion of edema - destructuring of muscle fibers, dictated by magnetic resonance imaging.
Re-injury (R)
It is a recurrence of the lesion, in a first episode it keeps a good prognosis while with a repetition, the condition worsens.

Source: own elaboration based on Futbol Club Barcelona Medical Services:An objective classification for muscle injuries, and Valle: Muscle Injuries in Sports ${ }^{12,13}$

\section{Innovation in the classification}

Currently, different sports entities have been commissioned to carry out research focused on a more optimal classification of muscle injuries, which allows a universal language (the most recent ones are based on ultrasound and magnetic resonance imaging), including the FC Barcelona medical team, which has started a new category for the interpretation of muscle injuries, allowing for a more objective approach. ${ }^{12}$ The use of this new classification allows to avoid the ambiguity of the interpretation and to give it a greater specificity of the presented table (Table 9). The expanded version allows for

Table 9 Muscle classification system

\begin{tabular}{|c|c|c|c|c|}
\hline \multicolumn{2}{|c|}{ Mechanism (M) } & Location (L) & Degree of severity (G) & Re-injury (R) \\
\hline \multirow{3}{*}{\multicolumn{2}{|c|}{ Direct Contusion $(\mathrm{T})$}} & P: proximal third of the muscular belly & $0-3$ & 0 : first episode \\
\hline & & M: middle third of the muscular belly & & $\mathrm{I}$ : first recurrence \\
\hline & & D: distal third of muscle belly & & 2: second recurrence or more \\
\hline \multirow{4}{*}{$\begin{array}{l}\text { Indirect } \\
\text { Contusion } \\
\text { (l) }\end{array}$} & & & & \\
\hline & Subtype & P: proximal third of the muscular belly & $0-3$ & 0: first episode \\
\hline & S: stretch & M: middle third of the muscular belly & & $\mathrm{I}$ : first recurrence \\
\hline & Q: sprint & D: distal third of muscle belly & & 2: second recurrence or more \\
\hline \multirow[t]{3}{*}{$\begin{array}{l}\text { No obvious } \\
\text { injury }(\mathrm{N})\end{array}$} & Subtype & Np: proximal third of the muscular belly & $0-3$ & 0 : first episode \\
\hline & S: stretch & $\mathrm{Nm}$ : middle third of the muscular belly & & $\mathrm{I}$ : first recurrence \\
\hline & Q: sprint & $\mathrm{Nd}$ : distal third of the muscular belly & & 2: second recurrence or more \\
\hline \multirow[t]{6}{*}{ Grade } & 0 & \multicolumn{3}{|c|}{ - Coding of indirect lesion with suggestive but negative clinical results on MRI, grade 0 indicated } \\
\hline & & \multicolumn{3}{|c|}{ - In some cases, the second letter describes the location of pain in the muscular belly } \\
\hline & one & \multicolumn{3}{|c|}{$\begin{array}{l}\text { - Hyperintense muscle fiber edema without intramuscular hemorrhage or architecture distortion (fiber architecture } \\
\text { and preserved angle of penetration) }\end{array}$} \\
\hline & & \multicolumn{3}{|c|}{ - Edema pattern: interstitial hyperintensity with feathery distribution in FSPD or T2 FSE + STIR image. } \\
\hline & 2 & \multicolumn{3}{|c|}{$\begin{array}{l}\text { - Muscle fiber hyperintensity - edema at the periphery of the tendon with minor distortion of the muscle fiber } \\
\text { architecture (poor fiber clarity and / or penetration angle distortion) } \pm \text { minor intermuscular hemorrhage, but no } \\
\text { quantifiable space between fibers. }\end{array}$} \\
\hline & & \multicolumn{3}{|l|}{ - Edema pattern, same as for grade I } \\
\hline
\end{tabular}

greater specificity because the letters of each category allow a more exact location of the lesion, as well as a more precise description structure for the table presented, without ambiguity or subjectivity of operation (Table 10). ${ }^{12,13}$ The muscular classification system allows a better reading of muscular injuries, offering a unique and simple nomenclature, which unifies various concepts in the medical field; It facilitates the proper approach of a patient with a muscle injury and that this is not misinterpreted, and therefore his recovery is optimal and fast. It should be noted that this system has not yet been used in controlled clinical studies and therefore not validated, despite being implemented in the sports community (Table 10)., ${ }^{911,12,14}$ 
Table Continued...

3

-Any quantifiable space between fibers in cranial-caudal or axial planes.

- Hyperintense focal defect with partial retraction of muscle fibers \pm intermuscular hemorrhage.

-The gap between the fibers in the maximum area of injury should be documented in an axial plane of the affected muscle belly.

-The exact percentage of CSA should be documented as a subscript of the rating

-When encoding an intra-tendon injury or an injury involving the MTJ or intramuscular tendon showing interruption / retraction or loss of tension (gap), a superscript ( $r$ ) must be added to the degree

Explanatory notes:

Penetration angle: site of insertion of muscle fibers

In indirect contusion: the lesion receives a subscript for the description if it is proximal or distal to the MTJ respectively

In no apparent injury $(\mathrm{N})$ à Refers to negative by magnetic resonance imaging, with subscripts

Abbreviations:

CSA: cross-sectional area

MRI: magnetic resonance imaging

FSE: fast spin echo

FSPD: Fat Density of Saturated Protons

MTJ: myotendinous junction

STIR: Short recovery of tau investment

Source: own elaboration based on Valle: Muscle Injuries in Sports (I3)

Table 10 Complications of muscle injuries

\begin{tabular}{|c|c|}
\hline Muscle Scar & $\begin{array}{l}\text { Retractable fibrous type scar, characteristic of a scar not properly treated, which predisposes to areas of anatomical } \\
\text { transition that may incur a re-tear of the lesion. By having an early and adequate therapy, a higher degree of } \\
\text { regeneration is obtained, which reduces the areas of scarring. An ultrasound image denotes a stellated, retractable } \\
\text { figure with decreased volume - muscle atrophy at the periphery of the scar }\end{array}$ \\
\hline Muscular hernia & $\begin{array}{l}\text { It constitutes a compromise of the muscular fascia due to a protrusion of a part of the muscle through a defect in it. } \\
\text { It tends to be asymptomatic in most cases although it can present pain or edema in the herniated area. By excellence, } \\
\text { ultrasound is used during rest and dynamically, to locate the size of the fascio defect and the extruded muscular } \\
\text { proportion }\end{array}$ \\
\hline $\begin{array}{l}\text { Muscle Pain } \\
\text { of Delayed } \\
\text { Appearance } \\
\text { DOMS }\end{array}$ & $\begin{array}{l}\text { It consists of the appearance of muscle pain for hours or days after physical activity or strenuous exercise, even } \\
\text { unaccustomed with a duration of } 5-7 \text { days and self-limited, which ultrasound shows an increase in volume of the } \\
\text { affected muscle with an important association to diffuse increase in echogenicity due to edema, and does not generally } \\
\text { require MRI }\end{array}$ \\
\hline $\begin{array}{l}\text { Compartment } \\
\text { syndrome }\end{array}$ & $\begin{array}{l}\text { Condition characterized by increased pressure (venous obstruction and muscular and nervous ischemia that lead } \\
\text { to necrosis) in a space confined by the anatomy that irreversibly damages its content and multiple causes. In the } \\
\text { ultrasound study, a significant increase in muscle volume with bulged fascia and loss of the normal fibrillar pattern is } \\
\text { observed, while on magnetic resonance imaging, swelling of the affected limb and alteration of the signal in the muscles } \\
\text { of the compartment are found. myonecrosis }\end{array}$ \\
\hline $\begin{array}{l}\text { Oscillating } \\
\text { Myositis }\end{array}$ & $\begin{array}{l}\text { Contusions with intramuscular bruises that can calcify and then ossify. It occurs frequently in those who practice } \\
\text { contact sports, especially in the muscle and pelvic regions, where calcifications are observed adjacent to the cortical } \\
\text { bone, which can be mistaken for a tumor.When presenting the clinical picture, there is chronic pain (indication for } \\
\text { surgical removal), with an evolution of months and a physical mass is felt on soft tissue upon physical examination. }\end{array}$ \\
\hline
\end{tabular}

Source: own elaboration based on Soto Ultrasound Classification of Muscle Tears and Muñoz: Sports Muscle Injuries ${ }^{3,9,10}$

\section{Complications}

Among the complications that can occur in muscle injuries (depending on their severity and complexity) can be found muscle scar, muscle hernia, late-onset muscle pain (some do not consider it a complete complication), compartment syndrome and myositis ossificans (Table 10). ${ }^{3,9,10}$

\section{Treatment - recovery}

Muscle tears or injuries heal between 3-16 weeks according to the magnitude of the injury and is mediated by the capacity for muscle regeneration - fibrous scarring, so treatment is based on stimulating regeneration and competition with scarring (which is not elastic and predisposing to other injuries). In this phase, ultrasound plays an important role in monitoring the healing process, where the initial cavity occupied by a hematoma gradually begins to fill from its periphery with granulatory tissue (visualized as confluent echogenic foci that end up occupying it completely). ${ }^{3,7,9,10}$ The management of muscle injuries is very simple but it must be taken into account for the proper performance of both its diagnosis and its therapeutic management. Lesions classified as mild can heal spontaneously and in a relatively short period of time, while those of a higher degree will require elements for their total restoration..$^{3,7,9,10,15-17}$ Current therapy includes rest (especially for bruising, use of cold and compression therapy (use of a compression bandage for the purpose of pain control as well as the possible extension of the injury and to allow the diffusion of fluids), therapy physical - kinesiotherapy (therapeutic and targeted exercises), and in some cases drugs (NSAIDs, pain relievers and muscle relaxants. In some cases, the use of enzyme injections or the surgical technique of washing liquid extraction is accepted; but it 
should be avoided to how massages on the area give rise as well as the application of heat, since this favors the extension as well as the inflation of the affected area. $3,7,9,10,15-17$

In addition to this therapy, there is the use of platelet-rich plasma (PRP), which aims to use growth factors and platelet-released cytokines to stimulate the repair process of a muscle injury, allowing multiple elite athletes being out of competition for a relatively short period of time. Caution in this type of therapy is the basis of these factors, which are fibrinogenic so they can be excessively harmful, due to the generation of more fibrocyte tissue and a risk of recurrence higher than natural; however, there is no consensus within the medical community regarding its use. ${ }^{3,7,9,10,15-17}$ The return of an athlete to their sport must be supported by various pillars such as strength-flexibility (pre-post-injury isokinetic tests, which are included in the athlete's file), imaging technique (RMI - US), tests functional on the affected muscle or muscles (mainly electromyography) and a strategic plan to reduce the risk of a recurrence or even avoid it entirely. ${ }_{3,7,9,10,15-17}$

\section{Conclusion}

Muscle injuries tend to be more frequent acute - chronic symptoms due to the change in lifestyles with the implementation of physical activity and even the desire to perform a competitive sport, for which there is a higher risk of the appearance of the same. Knowing how they develop, risk factors that can influence both presentation and recovery allows a better approach to them. The healing model is divided into 3 phases: destruction, repair and remodeling, all 3 well defined. The destruction phase occurs in the first days, and presents degeneration and active inflammation, causing a breakdown of muscle tissue and necrosis of the myofibrils for the formation of hematoma between them, as well as an inflammatory cell reaction. It is followed by the repair phase, where the necrotic tissue is reabsorbed, with regeneration of the myofibrils and production of an area of connective tissue and neovascularity; This phase occurs between 7-10 days post injury, with a peak after two weeks and a drop between 3-4 weeks. It ends with the remodeling phase, where there is a maturation of the neo myofibrils, a reorganization of the tissue that allows the muscular contractile capacity to be recovered. Once the process and its phases are known, the medical personnel and even the patient himself can know the evolution in the recovery as well as the lapses, means to use for its approach and resolution and even obtain better results by following the plan with understanding of it. Even if both parties are aware of imaging techniques, it is easier to determine the type of injury, its risks, possible complications and specific treatment (medical - pharmacological). Imaging techniques currently have various nomenclatures, which come from different points of view but upon reaching a consensus in the field, a better reading is allowed and therefore a better resolution of the picture of an athlete patient as well as an athlete of elite that allows you to reduce both risks and an increase in benefits for the return of the practice of sport. In other words, the use of general nomenclatures allows an approach aimed at a prompt resolution and restoration of the injury, as well as sports activity, so the use of ultrasound and magnetic resonance imaging, optimally and appropriately, is essential, as well as that anatomical knowledge and interpretation of the findings, for a prompt and effective evolution.

\section{Acknowledgments}

None.

\section{Conflicts of interest}

The autor declares there is no conflict of interest.

\section{References}

1. Guyton, Hall. Unit II: Physiology of the membrane, nerve and muscle. Treatise on Medical Physiology. 13 ${ }^{\text {th }}$ ed. Barcelona: Elsiever, 2016. p. 202-212.

2. Moore K, Dalley A, Agur A. Chapter 1: Overview and Basic Concepts. $8^{\text {th }}$ ed. Barcelona: Wolters Kluwer, 2017. p. 58-190.

3. Soto P, Salazar L. Ultrasonic Classification of Muscle Tears. Annals of Radiology Mexico. 2008:121-128.

4. Mansilla G. Muscle tears. MEDS Sports Medicine Clinic. Injuries and diseases: Article: Muscle Tears. 2013.

5. Fernández, N. Physiology laboratory manual: Practice 11: Muscle contraction. $6^{\text {th }}$ edition. Mexico: McGraw-Hill Interamericana. 2015.

6. Alfredo Elizondo Montemayor. Med Courses Muscle Contraction Physiology Guyton explained easy. Excitable tissue: muscle. Consulting in Health Sciences. 2018.

7. Pedreta C, Balius P. Muscle injuries in sport: An update from an article by Dr. Cabot, published in Notes on Sports Medicine in 1965. Apunts Med Esport. 2015;50(187):111-120.

8. Executioner MA. Ultrasonographic classification of muscle tears. Rev Chil Radiol. 2004;10:53-57.

9. Muñoz S., et al. Sports muscle injuries: Correlation between anatomy and imaging study. Rev Chil Radiol. 2017;23(3):164-175.

10. Muñoz S. Sports muscle injuries: Diagnostic imaging. Rev Chil Radiol. 2002;8:127-132.

11. Soccer medical services, Club Barcelona. Clinical practice guide for muscle injuries. Epidemiology, diagnosis, treatment and prevention. Version 4.5. Apunts Med Esport. 2009;164:179-203.

12. Barça Innovation Hub. An objective classification for muscle injuries. Sports performance, collective sports. 2018.

13. Valle X, Alentorn-Geli E, Tol JL, et al. Muscle Injuries in Sports: A New Evidence-Informed and Expert Consensus-Based Classification with Clinical Application. Sports Med. 2017;47:1241.

14. Dimmick S, Linklater J. Imaging of Acute Hamstring Muscle Strain Injuries. Semin Musculoskelet Radiol. 2017;21:415-432.

15. Medical Commission of the South American Soccer Confederation. Conmebol: Medical Commission and Anti-Doping Unit. 2017.

16. Rosas R. Sports Injuries: Clinic and Treatment. 2011;30(3).

17. Agustín Rubén Molina Rómoli, Luciano Andrés Rossi, Agustín Bernardo Bertona Altieri, et al. Platelet Rich Plasma For the Treatment of Acute Muscle Tears: Time to Return to Sport and Recurrence Rate. Randomized Controlled Study. Rev Asoc Argent Traumatol Sport. 2015;22(1). 